\title{
Mitteilungen der GI im Informatik Spektrum 3/2020
}

Online publiziert: 5. Mai 2020

๑) Springer-Verlag GmbH Deutschland, ein Teil von Springer Nature 2020

\section{Aus Vorstand und Präsidium}

\section{Vorankündigung: Einladung zur Ordentlichen Mitgliederversammlung der Gesellschaft für Informatik e.V. (GI)}

Sehr geehrte Damen und Herren,

hiermit lade ich Sie herzlich ein zur Ordentlichen Mitgliederversammlung der Gesellschaft für Informatik e. V. (GI). Sie findet statt am späten Nachmittag des 29. September 2020 im Kongresszentrum Karlsruhe. Details finden Sie auf der Webseite https://informatik2020.de/ und unter https://gi. de/.

\section{Tagesordnung}

1. Begrüßung

2. Bericht des Vorstandes über das abgelaufene Geschäftsjahr

1. Bericht des Präsidenten

2. Jahresabrechnung ${ }^{1}$

3. Bericht der Rechnungsprüfer für das Jahr 2019 und Entlastung von Vorstand, erweitertem Vorstand, Präsidium und Geschäftsführung

4. Wahl der Mitglieder der Rechnungsprüfungskommission für das Jahr 2020

5. Entgegennahme des Wirtschaftsplanes 2021

6. Bestätigung der Mitgliedsbeiträge 2021

7. Bericht der Kandidatenfindungskommission und Feststellung der endgültigen Kandidat/inn/enliste (Präsidiumsämter) für die Wahl 2020²

8. Bestimmung der Kandidatenfindungskommission für die Präsidiumswahl 2021

9. Wahl des Wahlausschusses für die Wahl 2020

\footnotetext{
1 Jahresrechnung und Wirtschaftsplan online. Die Jahresrechnung sowie den Wirtschaftsplan für das kommende Jahr finden Sie ab Juli unter gi.de im Mitgliederbereich.

${ }^{2}$ Die vorläufige Kandidatenliste finden Sie ab Juli unter gi.de im Mitgliederbereich.
}

10. Festlegung von Ort und Zeit für die Ordentliche Mitgliederversammlung 2022

11. Stellungnahme zu Anträgen auf Satzungsänderung

12. Genehmigung des Beschlussprotokolls der OMV 2020

13. Berichte

14. Verschiedenes

Bonn, im Juni 2020

Prof. Dr. Hannes Federrath, Präsident der Gesellschaft für Informatik e. V. (GI)

\section{Presse- und Öffentlichkeitsarbeit der GI \\ \#MINTatHome-Challenge: GI eröffnet Social-Media- Klassenzimmer (03.04.2020)}

Unter dem Dach von „Wir bleiben schlau! Die Allianz für MINT-Bildung zu Hause“ gestaltet die Gesellschaft für Informatik e. V. (GI) zusammen mit dem Bundesministerium für Bildung und Forschung (BMBF) mit der „\#MINTatHome-Challenge“ ein „Social-Media-Klassenzimmer“ für alle.

Der Schulausfall in der aktuellen Corona-Krise stellt Schülerinnen und Schüler, Lehrkräfte und Eltern vor neuen Herausforderungen. Gefördert vom Bundesministerium für Bildung und Forschung (BMBF) und unter dem Dach der „Wir bleiben schlau! Die Allianz für MINT-Bildung zu Hause" startet die Gesellschaft für Informatik e. V. eine Social-Media-Aktion für die schulfreie Zeit: Die „\#MINTatHome-Challenge“. Die Kampagne, die mit einem breiten Netzwerk an Partnern aus dem MINT-Bereich umgesetzt wird, soll Kindern und Jugendlichen aller Altersklassen spannende Knobelaufgaben aus der MINT-Welt online über soziale Netzwerke bereitstellen. Die Aktion startete am 3. April und ist zunächst bis zum Digitaltag am 19. Juni 2020 geplant. 


\section{Team der DHBW Karlsruhe gewinnt informatiCup $2020(27.03 .2020)$}

In der online übertragenen Endrunde der vier besten Hochschulteams des 15. informatiCups, des Studierenden-Wettbewerbs der Gesellschaft für Informatik e. V. (GI), setzte sich die DHBW Karlsruhe erfolgreich gegen die Teams der TU Braunschweig, Universität Oldenburg und RWTH Aachen durch.

In diesem Jahr ist alles anders: Die Endrunde des informatiCup 2020 der Gesellschaft für Informatik fand aufgrund der Corona-Pandemie als virtuelle Endrunde statt und wurde live im Internet übertragen. Der Hochschulwettbewerb, an dem dieses Jahr 56 Studierenden-Teams von bis zu vier Personen teilgenommen haben, hat angesichts der laufenden COVID-19-Krise eine ungeahnte Aktualität bekommen: Aufgabe war es, möglichst effektiv die Ausbreitung fiktiver Krankheitserreger in einem globalen Netzwerk von Städten zu verhindern. Im Zeitraum 01. November 2019-19. Januar 2020 wurden rund um diese Problemstellung eine Rekordzahl von qualitativ sehr hochwertigen Konzepten eingereicht, aus denen eine 14-köpfige Expertenjury ihre Auswahl zur Teilnahme an der Endrunde getroffen hat. Der Tag lässt sich auch im Nachhinein noch nacherleben unter https://www.youtube.com/watch? $\mathrm{v}=\mathrm{J} 2 \mathrm{G} 27 \mathrm{otDZTw}$.

Den kompletten Text der Pressemitteilungen finden Sie unter https://gi.de/aktuelles/presse.

\section{Aus den GI-Gliederungen}

\section{GI beim Empfang des Bundespräsidenten für den Deutschen Frauenrat}

Am 6. März empfing Bundespräsident Frank-Walter Steinmeier den Deutschen Frauenrat mit rund 120 Spitzenfrauen aus den Mitgliedsverbänden im Schloss Bellevue anlässlich des Internationalen Frauentags am 8. März. Die Fachgruppe Frauen und Informatik in der Gesellschaft für Informatik ist seit vielen Jahren Mitglied im Deutschen Frauenrat. Das Leitungsteam der Fachgruppe, Dr. Ursula Köhler und Sabine Kruspig, nahmen am Empfang teil.

Der Bundespräsident würdigte in seiner Rede das langjährige Engagement des Deutschen Frauenrates für Frauenrechte und lobte den Dachverband und seine Mitgliedsorganisationen als wichtige Schrittmacher für die Gleichberechtigung. Trotzdem seien die Errungenschaften der Frauenbewegung heute wieder bedroht. Die Demokratie werde ihren eigenen Idealen von Freiheit und Gleichheit erst dann gerecht, wenn Frauen und Männer gleichermaßen an ihr beteiligt seien, erinnerte Steinmeier.

Mona Küppers, die Vorsitzende des Deutschen Frauenrats, freute sich, dass der Deutsche Frauenrat zum ersten Mal in seiner fast 70-jährigen Geschichte vom Bundespräsidenten empfangen wurde (siehe Abb. 1). Sie betonte in ihrer Rede, dass die gleichberechtigte Teilhabe von Frauen mehr als eine demokratische Selbstverständlichkeit sei: sie ist ein Akt der politischen und ökonomischen Vernunft.

Im Anschluss an die Reden informierten sich FrankWalter Steinmeier und seine Frau Elke Büdenbender an
Abb. 1 Empfang des Bundespräsidenten für den Deutschen Frauenrat. (Quelle: Bundesregierung)

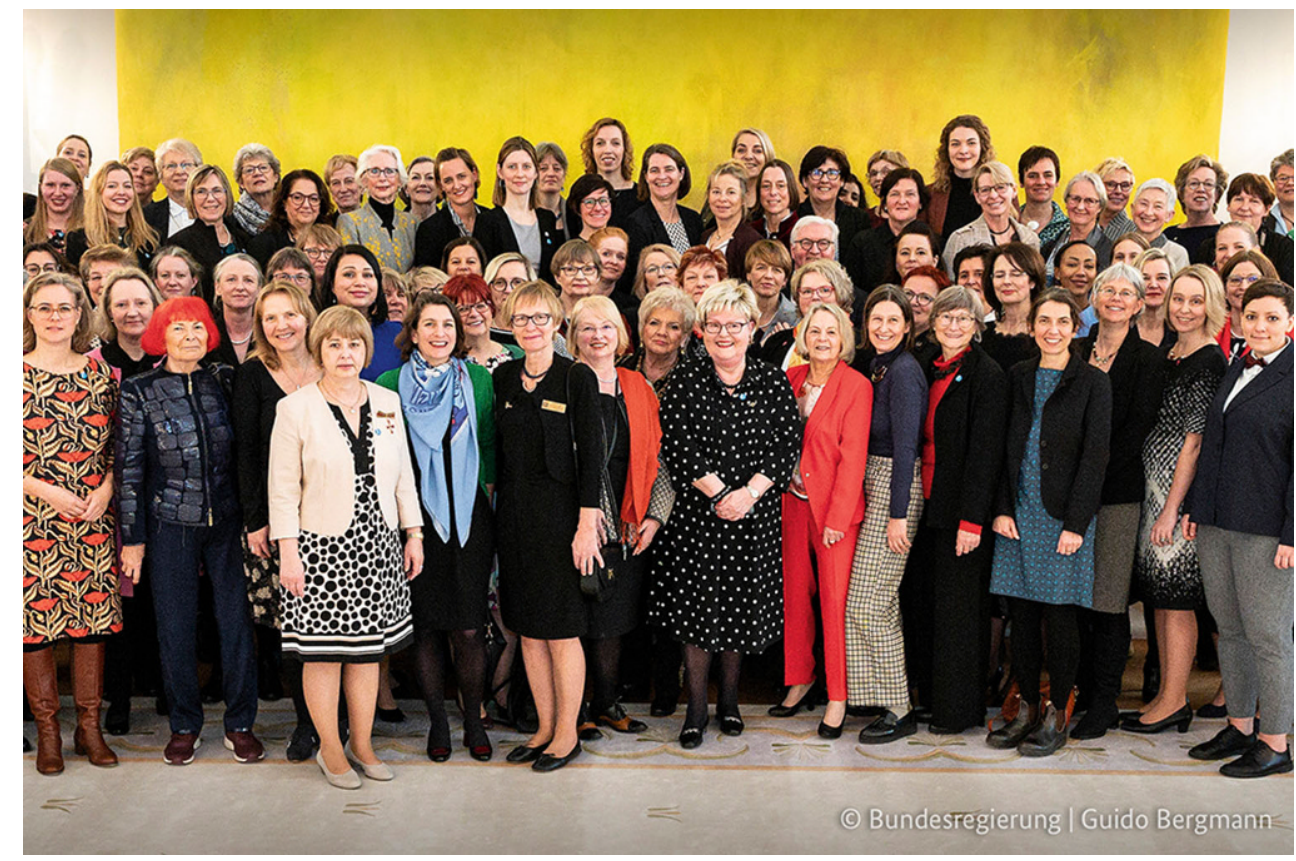


Thementischen zu den Arbeitsschwerpunkten ,Demokratie unter Druck“ und „Digitalisierung“. Zum Thema Digitalisierung gab es bereits von 2017-2019 einen Fachausschuss, in dem auch die Sprecherin der Fachgruppe mitgearbeitet hat. Das resultierende Positionspapier „Zukunft gestalten“ liegt in gedruckter Form vor und kann hier nachgelesen werden: https://www.frauenrat.de/unser-neues-positionspapierzur-digitalen-zukunft/.

\section{Neuer Arbeitskreis „Testen und KI“ in der Fachgruppe TAV des Fachbereichs Softwaretechnik}

Die Fachgruppe TAV (Test, Analyse und Verifikation von Software) des Fachbereichs Softwaretechnik der Gesellschaft für Informatik e. V. gründet den neuen Arbeitskreis „Testen und KI“ (TKI). Ein erstes Vortreffen interessierter TAV-Mitglieder erfolgte bereits im Februar 2019 auf dem 43. Treffen der FG TAV in Bremerhaven. Auf dem 44. FGTAV-Treffen in Stuttgart wurde dann die Gründung des Arbeitskreises beschlossen. Die beiden Themenschwerpunkte des Arbeitskreises TKI sind das Testen von KI-Systemen und die Nutzung von KI-Systemen zur Unterstützung des Testens. Dabei wird eine enge Zusammenarbeit mit den Fachgruppen des Fachbereichs „Künstliche Intelligenz“ (FB KI) der GI und anderen interessierten Organisationen angestrebt. Kommissarischer Sprecher des Arbeitskreises ist Prof. Dr. Mario Winter von der TH Köln, stellv. Sprecherin ist Prof. Dr. Bettina Buth von der HAW Hamburg. Bei Interesse melden Sie sich bitte bei mario.winter@thkoeln.de.

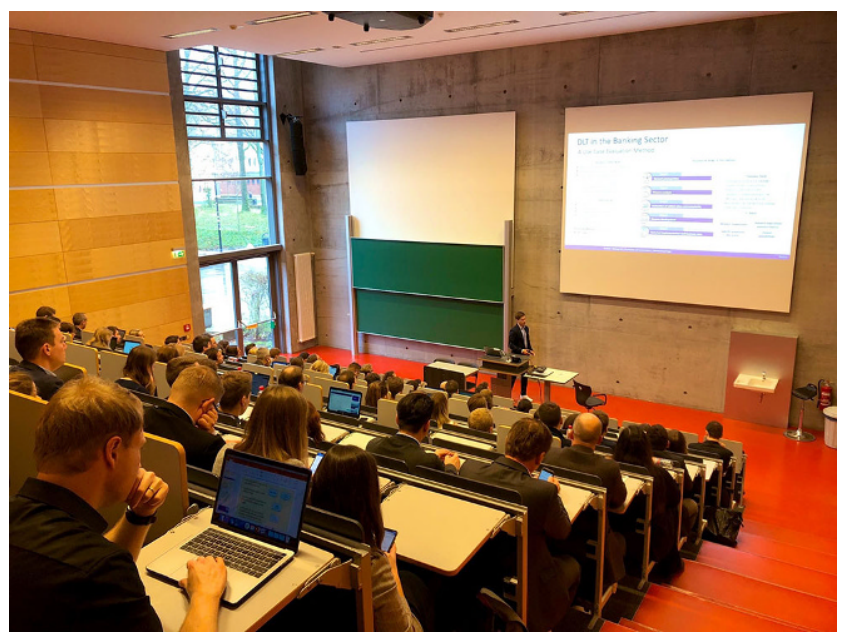

Abb. 2 Teilnehmerinnen und Teilnehmer der WI-Konferenz in Potsdam. (Foto: Universität Potsdam)

\section{Tagungsberichte}

\section{Internationale Tagung Wirtschaftsinformatik in Potsdam}

Vom 09.-11.03.2020 fand die 15. Internationale Tagung Wirtschaftsinformatik (WI 2020) trotz der herausfordernden Covid19-Situation an der Universität Potsdam statt (siehe Abb. 2). Die WI-Konferenz ist die größte Konferenz der Wirtschaftsinformatik im deutschsprachigen Raum und findet jährlich statt. Insgesamt 340 Teilnehmerinnen und Teilnehmer diskutierten in diesem Jahr aktuelle Themen und Herausforderungen der Wirtschaftsinformatik und ihrer Teildisziplinen in einer Mischung aus Präsenz- und Onlineformaten. Die drei Keynotes beleuchteten das Motto der Konferenz „Changing Landscapes-Shaping Digital Transformation and its Impact" aus den unterschiedlichen Perspektiven Ethik, Geschäftsmodelle sowie gesellschaftlicher Diskurs. Die Beiträge der insgesamt 24 Tracks sind kostenfrei online verfügbar (https://wi2020.de/de/proceedings).

Neben dem wissenschaftlichen Diskurs fanden im Rahmen der Konferenz ebenfalls unterschiedliche Sitzungen statt. So tagte beispielsweise das Leitungsgremium des Fachbereich Wirtschaftsinformatik der GI. Ein ansprechendes Rahmenprogramm rundete die Konferenz ab, die nächste WI findet 2021 in Duisburg-Essen statt.

Dr. Christof Thim, Universität Potsdam

\section{Tagungsankündigungen}

\section{Workshop „Methoden und Anwendungen der Computational Humanities"}

Zum nunmehr dritten Mal wird von der Fachgruppe „Informatik und Digital Humanities“ (InfDH) im Rahmen der INFORMATIK 2020 (Karlsruhe) ein Workshop veranstaltet. In diesem Jahr greifen wir eine aktuelle Entwicklung innerhalb der Digital Humanities-Community auf, bei der sich unter dem Schlagwort der „Computational Humanities“ in zunehmendem Maße ein eigener Teilbereich entwickelt, der primär auf statistische und algorithmische Analyseverfahren in den Geistes- und Kulturwissenschaften abzielt. „Computational Humanities“ bedeutet dabei im Wesentlichen eine Spezialisierung und Profilierung innerhalb des „,big tent“ Digital Humanities, dessen Spektrum von Digitalisierungs- und Modellierungsverfahren, digitalen Ansätzen für das Publizieren, Kommunizieren und Lehren bis hin zur Beschäftigung mit digitalen Kulturphänomenen (bspw. Computerspiele und eBooks) reicht.

Im geplanten Workshop wollen wir herausarbeiten, welche Beiträge die Informatik zum Feld der Computational Humanities leisten kann. Eingeladen sind Beiträge, die ex- 
plizit die Entwicklung, Anwendung oder Evaluation von Methoden und Verfahren aus dem Spektrum der Computational Humanities zum Thema haben. Bei allen Beiträgen sollte der Aspekt der quantitativen, computergestützten Analyse geistes- und kulturwissenschaftlicher Daten herausgearbeitet werden. Nähere Informationen finden sich unter https://fg-infdh.gi.de/infdh-worskshop-2020.

\section{Bundesweite Informatikwettbewerbe (BWINF)}

\section{Teilnahmerekord beim Jugendwettbewerb Informatik - Zusatzangebot wegen Schulschließungen}

Mehr als 20.000 Schülerinnen und Schüler und damit so viele wie nie haben vom 24. Februar bis 8. März 2020 an der 1. Runde des Jugendwettbewerbs Informatik (JwInf) teilgenommen. Die Mädchenquote blieb konstant bei $32 \%$. Die Teilnahmezahl hat sich von 16.506 im letzten Jahr auf mehr als 20.000 in diesem Jahr gesteigert.

\section{Zusätzlicher Wettbewerbszeitraum für die 2. Runde}

Die Bundesweiten Informatikwettbewerbe reagierten auf die Schulschließungen mit einem Zusatzangebot. Nach den Osterferien gab es eine zusätzliche JwInf-Wettbewerbswoche, die eine Teilnahme im Klassenverband ermöglichen sollte. Ursprünglich erstreckte sich die 2. JwInf-Runde auf den Zeitraum 23. März bis 5. April 2020.

\section{Weitere Programmieraufgaben}

Für alle Schülerinnen und Schüler, die über den Wettbewerb hinaus noch Lust auf Programmier-Herausforderungen hatten, gab es seit Ende März weitere Wettbewerbsangebote unter jwinf.de. Diese bestanden überwiegend aus Aufgaben aus den vergangenen Wettbewerben, aber auch aus einigen neue Aufgaben.

Unter bwinf.de/corona wurden dringliche Fragen zum BWINF-Angebot beantwortet und auf Zusatzangebote für Schülerinnen und Schüler hingewiesen.

\section{Runde}

Die Gesamtpunktzahl der 1. und 2. Runde entscheidet über die Qualifikation für die 3. Runde. Die 3. Runde des JwInf startet zeitgleich mit dem 39. Bundeswettbewerb Informatik am Dienstag, 1. September 2020. Einsendeschluss ist der 23. November 2020.

\section{Tagungen}

\subsubsection{0-10.06.2020 - Berlin}

Languages \& the Media 2020 13th International Conference on Language Transfer in Audiovisual Media

LM2020

https://www.languages-media.com/

\subsubsection{0-09.06.2020 - Kloster Seeon}

European Conference of Software Engineering Education ECSEE 2020

http://www.ecsee.eu

\subsubsection{0-27.06.2020 - Regensburg}

34th Annual IFIP WG11.3 Conference on Data and Applications Security and Privacy

DBSec 2020

https://dbsec2020.ur.de

\subsubsection{0-19.08.2020 - Potsdam}

Informatik-Sommercamp für Schülerinnen und Schüler https://hpi.de/open-campus/schuelerakademie/schueler camps/hpi-sommercamp.html

\subsubsection{0-09.09.2020 - Magdeburg}

Tagung Mensch und Computer 2020

MuC2020

https://muc2020.mensch-und-computer.de

\subsubsection{0-11.09.2020 - Leipzig}

Digital Operating Room Summer School DORS

https://www.iccas.de/en/dors/

\subsubsection{0-25.09.2020 - Bamberg}

43rd German Conference on Artificial Intelligence KI 2020

https://ki2020.uni-bamberg.de/

\subsubsection{0-01.10.2020 - Karlsruhe}

50. Jahrestagung der Gesellschaft für Informatik

INFORMATIK 2020

https://informatik2020.de/ 


\subsubsection{0-30.10.2020 - Essen}

The 15th Workshop in Primary and Secondary Computing Education

WIPSCE 2020

https://www.wipsce.org/2020/

\subsubsection{0-03.12.2020 - Berlin}

9th International Conference on Performance Evaluation and Modeling in Wired and Wireless Networks PEMWN 2020

https://sites.google.com/view/pemwn2020/home

\subsubsection{1-26.02.2012 - Braunschweig}

Software Engineering 2021

SE 2021

https://se-2021.tu-bs.de/

\subsubsection{1-12.03.2021 - Dresden}

19. Fachtagung für Datenbanksysteme für Business, Technologie und Web

BTW 2021

http://btw-konferenz.de/2021

\subsubsection{1-10.09.2021 - Wuppertal}

GI-Fachtagung Informatik und Schule - Lehrerbildung INFOS 2021

http://www.infos2021.de/ 